\title{
Midbrain/pons area ratio and clinical features predict the prognosis of Progressive Supranuclear Palsy
}

shishuang cui

Shanghai Jiao Tong University Medical School Affiliated Ruijin Hospital huawei ling

Shanghai Jiao Tong University Medical School Affiliated Ruijin Hospital juanjuan du

Shanghai Jiao Tong University Medical School Affiliated Ruijin Hospital yiqi lin

Shanghai Jiao Tong University Medical School Affiliated Ruijin Hospital jing pan

Shanghai Jiao Tong University Medical School Affiliated Ruijin Hospital haiyan zhou

Shanghai Jiao Tong University Medical School Affiliated Ruijin Hospital gang wang

Shanghai Jiao Tong University Medical School Affiliated Ruijin Hospital ying wang

Shanghai Jiao Tong University Medical School Affiliated Ruijin Hospital qin xiao

Shanghai Jiao Tong University Medical School Affiliated Ruijin Hospital jun liu

Shanghai Jiao Tong University Medical School Affiliated Ruijin Hospital yuyan tan

Shanghai Jiao Tong University Medical School Affiliated Ruijin Hospital shengdi chen ( $\nabla$ chensd@rih.com.cn)

Shanghai Jiao Tong University Medical School Affiliated Ruijin Hospital

\section{Research article}

Keywords: Progressive Supranuclear Palsy, survival analysis, M/P area ratio

Posted Date: March 13th, 2020

DOI: https://doi.org/10.21203/rs.2.19508/v3 
License: (c) (i) This work is licensed under a Creative Commons Attribution 4.0 International License. Read Full License

Version of Record: A version of this preprint was published at BMC Neurology on March 30th, 2020. See the published version at https://doi.org/10.1186/s12883-020-01692-6. 


\section{Abstract}

Backgrounds: Progressive Supranuclear Palsy (PSP) is a rare movement disorder with poor prognosis. This retrospective study aims to characterize the natural history of PSP and to find predictors of shorter survival and faster decline of activity of daily living. Method: All patients recruited fulfilled the movement disorder society (MDS) clinical diagnostic criteria for PSP (MDS-PSP criteria) for probable and possible PSP with median 12 years. Data were obtained including age, sex, date of onset, age at onset (AAO), symptoms reported at first visit and follow-up, date of death and date of institutionalization. Magnetic resonance imaging was collected at the first visit. Endpoints were death and institutionalization. KaplanMeier method and Cox proportional hazard model were used to explore factors associated with early death and institutionalization. Results: 59 patients fulfilling MDS-PSP criteria were enrolled in our study. 19 patients (32.2\%) had died and 31 patients (52.5\%) were institutionalizedl by the end of the follow-up. Predictors associated with poorer survival were late-onset PSP and decreased M/P area ratio. Predictors associated with earlier institutionalization were older $A A O$ and decreased $M / P$ area ratio. Conclusion: older and decreased M/P area ratio were predictors for earlier dearth and institutionalization in PSP. The neuroimaging biomarker $\mathrm{M} / \mathrm{P}$ area ratio was a predictor for prognosis in PSP.

\section{Background}

Progressive Supranuclear Palsy (PSP) is a rare movement disorder with an estimated prevalence of 6.4 per 100000 , characterized by the accumulation of abnormally phosphorylated tau protein in the basal ganglia, frontal lobe and brainstem[1]. The cardinal clinical features of PSP are early postural instability, supranuclear gaze palsy, akinesia, cognitive impairment and behavior changes[2].

The prognosis of PSP is poor with increased risks of falls, dysphagia, aspiration pneumonia and pressure ulcer, leading to institutionalisation and short survival time[3]. The mean survival is only 3 to 4 years after diagnosis and 5.3 to 13.0 years at onset of disease.[1]. Previous studies indicated that male, older age at onset (AAO), early falls, dementia and clinical phenotypes were predictors of poor survival[1, 4-9].

Midbrain atrophy is commonly observed in PSP. A number of midbrain metrics including "hummingbird" sign, midbrain/pons (M/P) area ratio, magnetic resonance parkinsonism index (MRPI) have been proposed as potential neuroimaging biomarkers in PSP[10-13]. Neuroimaging biomarkers such as M/P area ratio are more objective and sensitive. It may provide more detailed information about progression of disease and therapeutic effects of anti-parkinsonism drugs, including levodopa. Thus, it is of value to examine the utility of neuroimaging biomarkers for disease prognosis. A previous study found that MRPI predicts vertical supranuclear gaze palsy in patients with PSP[14]. But no study has explored whether neuroimaging biomarker can predict prognosis of PSP. We hypothesize that M/P area ratio indicates a poor prognosis in PSP.

Additionally, previous studies mainly focused on survival rather than activity of daily living which reflects the progression of disability of living and is less influenced by factors such as nursing. 
This study aims to characterize the natural history of PSP and to find predictors including neuroimaging biomarkers of shorter survival and faster decline of activity of daily living.

\section{Methods}

\subsection{Participant}

Patients were recruited at Department of Neurology \& Institute of Neurology, Rui Jin Hospital Affiliated to Shanghai Jiao Tong University School of Medicine from 2005 to 2016. We enrolled patients fulfilling the Neurological Diseases and Stroke-Society for PSP (NINDS-SPSP) clinical diagnostic criteria and reconfirmed and corrected them with the movement disorder society (MDS) clinical diagnostic criteria for PSP (MDS-PSP criteria) for probable and possible PSP according to their disease history and information including neuroimaging[2]. The onset of disease of all patients should be before Jan 2015 to ensure that we can explore for presence of symptoms within 3 years of disease onset. The study was approved by the medical ethics committee of Rui Jin Hospital affiliated to Shanghai Jiao Tong University School of Medicine. Participants were given written informed consent before inclusion in the study.

\section{2 data collection}

Data was obtained from medical records or telephone interview including age, gender, date of onset, age at onset (AAO), interval from onset to brain MRI, side of onset, symptoms reported at first visit and followup, date of death and date of institutionalization. Information on the occurrence and time of onset of fall, freezing of gait (FOG), bulbar palsy, apathy and urinary incontinence was collected. Patients with symptoms mentioned above within 3 years of first symptom were defined as having an early onset (short latency) of those features[2, 8]. Patients were also assessed with Mini-Mental State Examination (MMSE) at the first visit.

Endpoints were death and institutionalization. Institutionalization was defined as Hohn-Yahr stage V. Disease duration to death was defined as interval from disease onset to death or to the end of follow-up (June 2018). Disease duration to institutionalization was defined as interval from disease onset to institutionalization or to the end of follow-up (June 2018).

According to MDS-PSP criteria, patients were subdivided into PSP with Richardson's syndrome (PSP-RS) and non-PSP-RS phenotype[2]. Non-PSP-RS phenotype includes PSP with predominant parkinsonism (PSP-P), PSP with predominant frontal presentation (PSP-F), PSP with progressive gait freezing (PSPPGF), PSP with predominant ocular motor dysfunction (PSP-OM), PSP with predominant speech/language disorder (PSP-SL)[2].

\subsection{MRI acquisition and measurement}

Non-contrast Brain MRI (GE, USA) was performed with 1.5-tesla MRI scanner included T1-weighted sagittal images, T1-weighted axial images and FLAIR axial images. 
The manual measurements were performed on midsagittal TI-weighted MRI with Mricro as shown in figure 1. The T1-weighted sagittal images were performed with echo time (TE) $9.09 \mathrm{~ms}$, repetition time (TR) $2510.96 \mathrm{~ms}$ and voxel size $0.47 * 0.47 * 6 \mathrm{~mm}^{3}$. The brainstem was divided into 3 sub-regions (midbrain, pons and medulla oblongata) on T1 midsagittal sections by drawing 2 cutting planes: the first between the superior pontine notch and inferior edge of the quadrigeminal plate (line1) and the second parallel to the first through the inferior pontine notch (line 2) (figure 1A) [10,12, 13, 15].

The midbrain area was above line 1 (excluding the tectum) (figure 1B). The pons area was between the liquor- parenchyma-border of the pons, the anterior liquor-parenchyma-border of the fourth ventricle, line 1 and line 2 (figure $1 \mathrm{C}$ ). M/P area ratio was calculated as the ratio of midbrain area to pons area.

Image analysis was performed by two independent raters. To assess the intrarater reliability, a second evaluation was made 1 week after the first evaluation by one of the two raters.

Figure 1

\subsection{Statistical analysis}

Demographic and clinical variables were presented as frequency or percentage for categorical variables, or as the mean and standard deviation for continuous variables. Intraclass correlation coefficient was used to assess the intrarater and interrater reliability. Survival curves for each categorical variable were estimated by the Kaplan-Meier (K-M) method and differences in survival were measured by the log rank test. The risk of death or institutionalization was calculated using the Cox proportional hazard model in univariate analysis; variables associated with significance were entered in the multiple stepwise regression model. The Variance Inflation Factor (VIF) of variables included in multivariate analysis were all below 5 . Hazard ratio (HR) and 95\% confidence interval were calculated. Significance was tested at the $5 \%$ level.

\section{Results}

59 patients fulfilling MDS-PSP criteria were enrolled in our study. Clinical and demographic characteristics of patients are summarized in table 1.43 (72.9\%) patients were classified as PSP-RS, while 16 (27.1\%) were classified as non-PSP-RS including 11 patients with PSP-P, 2 with PSP-F and 3 with PSP-FOG. 19 patients (32.2\%) had died and 31 patients (52.5\%) were institutionalized by the end of the follow-up. The median survival time was 12.0 years and median time from onset of the disease to institutionalization was 9.0 years.

The mean M/P area ratio in MRI was $21.3 \%$ (table 1). Correlation between intrarater for midbrain area was 0.991 and for pons area was 0,998. Correlation between interrater for midbrain area was 0.992 and for pons was 0.999 .

Stratified by gender, phenotype and presence or absence of clinical features within 3 years, we found that patients with early falls, early bulbar palsy, early apathy and early incontinence showed shorter survival 
time (log-rank: $p=0.032, p=0.013, p=0.018, p=0.001)$ compared to patients without this feature within 3 years. Patients with early bulbar palsy, early apathy, early incontinence showed shorter time from onset to institutionalization by K-M method $(p=0.016, p=0.027, p=0,003)$. In addition, a comparison between PSPRS and PSP-P excluding PSP-F and PSP-FOG was also performed. Compared with PSP-RS, PSP-P had a longer survival in trend (log-rank: $p=0.067$ ), but no significant difference was observed for institutionalization by K-M method (log-rank: $p=0.115)$.

In univariate analysis using the Cox proportional hazard model, early onset of falls, apathy, bulbar paralysis and urinary incontinence were associated with higher mortality risk (Table 2). Decreased M/P area ratio was associated with higher mortality risk after adjusting for age and duration from onset to visit. The selected multivariate regression model included gender, age at onset, early falls, early apathy, early bulbar palsy, early urinary incontinence and M/P area ratio adjusted by age. In this model, older age at onset and decreased $\mathrm{M} / \mathrm{P}$ area ratio were independently associated with higher mortality risk.

In univariate analysis using the Cox proportional hazard model, early onset of apathy, bulbar paralysis and urinary incontinence were associated with higher institutionalized risk (Table 3). Decreased M/P area ratio was associated with higher institutionalized risk after adjusting for age and duration from onset to visit. The selected multivariate regression model included gender, age at onset, early bulbar palsy, early apathy, early urinary incontinence and M/P area ratio adjusted by age. In this model, older age at onset and decreased M/P area ratio were independently associated with higher institutionalized risk.

\section{Discussion}

To the best of our knowledge, this paper is the first to consider the neuroimaging biomarker M/P area ratio as a predictor for prognosis and to explore the factors associated with the progression of disability of living. In this paper, we found that predictors associated with poorer survival and earlier institutionalization were older age at onset and decreased M/P area ratio.

In the survival analysis, decreased $\mathrm{M} / \mathrm{P}$ area ratio was associated with earlier death and institutionalization. Paviour et al found that M/P area ratio was smaller in PSP than Parkinson's disease or multiple system atrophy or controls[16]. The phenomenon that midbrain atrophy progressed in PSP-RS and PSP-P was observed in previous researches $[15,17]$. Previous studies found that brainstem atrophy rates, especially change in midbrain volume were correlated with disease progression measured by PSP Rating Scale[18-21]. Inability to move eyes downward early was observed to predict survival time and midbrain atrophy predicted the disease progression of supranuclear gaze palsy[14, 22]. These researches indicated that $\mathrm{M} / \mathrm{P}$ area ratio may be related to progression of motor disability and death risk in PSP. Axial symptoms may influence the survival time and it is also associated with pathological change in brainstem, especially in midbrain, thus they are associated with a smaller midbrain volume[16]. Besides, correlation were identified in previous studies between midbrain volume and motor disability and between PSPRS scores and [18F]AV-1451 uptake in midbrain, indicating that pathological change in midbrain 
might predict more severe disease[16, 23]. Thus, it might be useful as a progression marker in clinical trial for PSP.

In accordance with most previous studies, older age at onset is associated with shorter survival[6-9, 24]. But other few studies have found negative or inverse association between AAO and survival time[22, 25]. Shorter survival and institutionalization for people were expected for the elderly, regardless of disease[9]. In our study, we also observed older AAO was a predictor for early institutionalization, which means patients with older AAO is associated with more rapid progression in motor disability. Consistent with our results, Golb et al found that patients with older AAO has a higher PSP rating scale score and progressing more rapidly[24].

Few studies investigated the association between urinary incontinence and survival time, though it was one of the prevalent autonomic symptoms in PSP[25-27]. In our study, we found the association between incontinence and survival and institutionalization in univariate model, but the association became negative in multivariate model. Rare studies explored the association between apathy and prognosis in PSP despite that apathy is a common psychiatric symptom in PSP. In our study, we found that apathy was associated with death and institutionalization in earlier institutionalization in univariate model, but the association became negative in multivariate model. But the sample of our study was small, larger sample is needed to verify our results.

Inconsistent with previous studies found that RS phenotype was associated with a worse prognosis $[4,5$, 8], no significant association was found between phenotype and prognosis with K-M method or Cox proportional hazard model in our study. Reasons for the inconsistence we consider are as followings. Previous studies mostly compared PSP-RS phenotype versus PSP-P phenotype while non-RS phenotype explored in our study included PSP-P, PSP-F and PSP-FOG. Published studies found that the median survival time of patients with PSP-F was similar with PSP-RS and its cumulative mortality after 5 years was mildly higher than PSP-RS [28-30]. The dementia and frontal symptoms, the most common features in PSP-F, were associated with earlier death[26, 31]. A recently published study found cognitive impairment in PSP, especially executive dysfunction, was associated with severity of PSPrelated tau pathology in autopsy-confirmed PSP patients, which might explain the association with poorer prognosis[32]. After excluding PSP-F and PSP-FOG, we found that a trend for the association of PSP-P with a longer survival. The small number of our sample may also influence our results. Thus, a larger sample with various phenotype is needed for further exploration. Besides, the MDS-PSP criteria was used in this study, while previous studies using NINDS-SPSP. This difference of two diagnosis criteria may account for the negative results to some extent.

Furthermore, unlike in previously published articles, the criteria for diagnosis and classification in the study was MDS-PSP rather than NINDS-SPSP. Respondek et al found that the sensitivity of NINDS-SPSP to detect non-RS phenotype was relatively unsatisfactory[29]. MDS-PSP based on NINDS-SPSP added akinesia and cognitive dysfunction besides ocular motor dysfunction, postural instability as core symptoms, thus leading to the improved detection for non-RS phenotype[2]. 
The strength of our study are as follows: 1区the present study is the first one to investigate the association between neuroimaging and prognosis in PSP; 2 ) in addition to explore factors associated with early death, we also explored factors associated with early institutionalization to find out what factors influence progression of disability; 3) some non-motor symptoms including apathy and urinary incontinence rarely mentioned in previous studies were included in our research to explore their role on survival and progression in PSP; 4) MDS-PSP criteria was used in our study to contain a wider spectrum of PSP.

Our study has several limitations. First, this was a retrospective study based on medical history and interview. The information of medical history may be less accurate for inaccurate memory of patients and incomplete medical history interviewed by doctor. However, the information was completed by movement disorder specialists and reconfirmed by a structured interview with the patient or their caregivers, making it more accurate. Secondly, the sample in our study was relatively small. However, PSP is a rare disease per se. Third, the diagnosis was made by clinical criteria. However, the patients enrolled fulfilled the probable and possible PSP of PSP-MDS criteria which has relatively high specificity[2]. Besides, mean interval between onset of symptoms and MRI was 3,8 years, which was a long time, since atypical clinical features and structural neuroimaging findings may not appear in the early disease course, interfering the diagnosis of PSP. Thus, an early neuroimaging marker are needed.

\section{Conclusion}

Older $\mathrm{AAO}$ and decreased $\mathrm{M} / \mathrm{P}$ area ratio were predictors for earlier death and earlier institutionalization in PSP. The neuroimaging biomarker M/P area ratio was a predictor for prognosis in PSP. Prospective studies with larger sample size will be needed further to confirm the predictors for prognosis.

\section{Abbreviations}

$\mathrm{AAO}$, age at onset

FOG, freezing of gait

HR, Hazard ratio

M/P, midbrain/pons

MDS, the movement disorder society

MMSE, Mini-Mental State Examination

MRPI, magnetic resonance parkinsonism index

NINDS-SPSP, the Neurological Diseases and Stroke-Society for PSP 
PSP, Progressive Supranuclear Palsy

PSP-CBS, PSP with predominant corticobasal syndrome

PSP-F, PSP with predominant frontal presentation

PSP-OM, PSP with predominant ocular motor dysfunction

PSP-P, PSP with predominant parkinsonism

PSP-PGF, PSP with progressive gait freezing

PSP-RS, PSP with Richardson's syndrome

PSP-SL, PSP with predominant speech/language disorder

VIF, Variance Inflation Factor

\section{Declarations}

\section{Ethics approval and consent to participate}

The study was approved by the medical ethics committee of Rui Jin Hospital affiliated to Shanghai Jiao Tong University School of Medicine. Participants were given written informed consent before inclusion in the study.

\section{Consent to publish}

Not Applicable.

\section{Availability of data and materials}

All data generated or analysed during this study are included in this published article.

\section{Competing interests}

The authors declare that they have no competing interests.

\section{Funding}

This work was supported by grants from the National Natural Science Foundation of China [grant numbers 81430022, 81771374, 91332107, 81371407], Foundation of Shanghai Municipal Education Commission (2017NKX001) and Shanghai Sailing Program (19YF1429900). the National Natural Science Foundation of China was used to pay the fee of publish, language editing and print. Foundation of Shanghai Municipal Education Commission was used to pay the fee of researcher. Shanghai Sailing Program was used to pay the phone bill. 
CSS analyzed the data and drafted the article. LHW complete the MRI scan. DJJ and LYQ analysis the neuroimaging. PJ and ZHY designed the study and collect the information. WG and WY collect the information and revised the manuscript. XQ, LJ and TYY collected the information and analyzed the data. CSD conceptualized and revised the manuscript. All authors read and approved the final manuscript.

\section{Acknowledgments}

We are grateful to all the patients and their families who participated in this study. We also thank colleagues who performed the neuroimaging test.

\section{References}

1. Glasmacher SA, Leigh PN, Saha RA: Predictors of survival in progressive supranuclear palsy and multiple system atrophy: a systematic review and meta-analysis. J Neurol Neurosurg Psychiatry 2017, 88(5):402-411.

2. Hoglinger GU, Respondek G, Stamelou M, Kurz C, Josephs KA, Lang AE, Mollenhauer B, Muller U, Nilsson C, Whitwell JL et al: Clinical diagnosis of progressive supranuclear palsy: The movement disorder society criteria. Mov Disord 2017, 32(6):853-864.

3. Shea YF, Shum ACK, Lee SC, Chiu PKC, Leung KS, Kwan YK, Mok FCK, Chan FHW: Natural clinical course of progressive supranuclear palsy in Chinese patients in Hong Kong. Hong Kong Med J 2019, 25(6):444-452.

4. O'Sullivan SS, Massey LA, Williams DR, Silveira-Moriyama L, Kempster PA, Holton JL, Revesz T, Lees AJ: Clinical outcomes of progressive supranuclear palsy and multiple system atrophy. Brain 2008, 131(Pt 5):1362-1372.

5. Jecmenica-Lukic M, Petrovic IN, Pekmezovic T, Kostic VS: Clinical outcomes of two main variants of progressive supranuclear palsy and multiple system atrophy: a prospective natural history study. $J$ Neuro/ 2014, 261(8):1575-1583.

6. Chiu WZ, Kaat LD, Seelaar H, Rosso SM, Boon AJ, Kamphorst W, van Swieten JC: Survival in progressive supranuclear palsy and frontotemporal dementia. J Neurol Neurosurg Psychiatry 2010, 81(4):441-445.

7. U. Nath MYB-S, MD; R.G. Thomson, MD; A.J. Lees, MD; and D.J. Burn, MD: Clinical features and natural history of progressive supranuclear palsy A clinical cohort study. Neurology 2003, 60:910916.

8. dell'Aquila C, Zoccolella S, Cardinali V, de Mari M, Iliceto G, Tartaglione B, Lamberti P, Logroscino G: Predictors of survival in a series of clinically diagnosed progressive supranuclear palsy patients. Parkinsonism \& Related Disorders 2013, 19(11):980-985.

9. Papapetropoulos S, Gonzalez J, Mash DC: Natural History of Progressive Supranuclear Palsy: A Clinicopathologic Study from a Population of Brain Donors. European Neurology 2005, 54(1):1-9. 
10. Moller L, Kassubek J, Sudmeyer M, Hilker R, Hattingen E, Egger K, Amtage F, Pinkhardt EH, Respondek G, Stamelou M et al: Manual MRI morphometry in Parkinsonian syndromes. Mov Disord 2017, 32(5):778-782.

11. Whitwell JL, Hoglinger GU, Antonini A, Bordelon Y, Boxer AL, Colosimo C, van Eimeren T, Golbe LI, Kassubek J, Kurz C et al: Radiological biomarkers for diagnosis in PSP: Where are we and where do we need to be? Mov Disord 2017, 32(7):955-971.

12. Nigro S, Arabia G, Antonini A, Weis L, Marcante A, Tessitore A, Cirillo M, Tedeschi G, Zanigni S, Calandra-Buonaura G et al: Magnetic Resonance Parkinsonism Index: diagnostic accuracy of a fully automated algorithm in comparison with the manual measurement in a large Italian multicentre study in patients with progressive supranuclear palsy. Eur Radiol 2017, 27(6):2665-2675.

13. H. Oba MAY, MD; H. Terada, MD; A.J. Barkovich, MD; K. Kutomi, MD; T. Yamauchi, MD; S. Furui, MD; T. Shimizu, MD; M. Uchigata, MD; K. Matsumura, MD; M. Sonoo, MD; M. Sakai, MD; K. Takada, MD; A. Harasawa, MD; K. Takeshita, MD; H. Kohtake, MD; H. Tanaka, MD; and S. Suzuki, MD: New and reliable MRI diagnosis for progressive supranuclear palsy Neurology 2005, 64:2050-2055.

14. Aldo Quattrone MMM, MD David R. Williams, PhD,FRACP Basilio Vescio, PhD Gennarina Arabia, MD, MSc Salvatore Nigro, PhD Giuseppe Nicoletti, MD Maria Salsone, MD Fabiana Novellino, MD Rita Nisticò, MD Franco Pucci, PhD Carmelina Chiriaco, PhD Pierfrancesco Pugliese,, MD Domenico Bosco MMC, MD: MR parkinsonism index predicts vertical supranuclear gaze palsy in patients with PSP-parkinsonism. Neurology 2016, 87:1-8.

15. Hwang M, Yang H, Kim Y, Youn J, Park J, Huh YE, Kim H-T, Cho JW: Differential Progression of Midbrain Atrophy in Parkinsonism: Longitudinal MRI Study. Neurodegenerative Diseases 2017, 17(1):31-37.

16. Paviour DC, Price SL, Jahanshahi M, Lees AJ, Fox NC: Regional brain volumes distinguish PSP, MSAP, and PD: MRI-based clinico-radiological correlations. Mov Disord 2006, 21(7):989-996.

17. Agosta F, Caso F, Jecmenica-Lukic M, Petrovic IN, Valsasina P, Meani A, Copetti M, Kostic VS, Filippi M: Tracking brain damage in progressive supranuclear palsy: a longitudinal MRI study. J Neurol Neurosurg Psychiatry 2018, 89(7):696-701.

18. Shubir Dutt B, Richard J. Binney, PhD Hilary W. Heuer, PhD Phi Luong, BS, Suneth Attygalle, MS Priyanka Bhatt, MS Gabe A. Marx, BA Jonathan Elofson, BS Maria C. Tartaglia, MD Irene Litvan, MD, Scott M. McGinnis MBCD, MD JK, PhD, Dana Waltzman, PhD Lisa Voltarelli, BS Norbert Schuff, PhD, Gil D. Rabinovici, MD Joel H. Kramer, PsyD Clifford R. Jack, Jr., MD Bruce L. Miller, MD Howard J. Rosen, MD Adam L. Boxer, MD,PhD,On behalf of the AL-108-231 investigators: Progression of brain atrophy in PSP and CBS over 6 months and 1 year. Neurology 2016, 87:2016-2025.

19. Tsai RM, Lobach I, Bang J, Whitwell JL, Senjem ML, Jack CR, Jr., Rosen H, Miller B, Boxer AL, Investigators AL: Clinical correlates of longitudinal brain atrophy in progressive supranuclear palsy. Parkinsonism Relat Disord 2016, 28:29-35.

20. Whitwell JL, Tosakulwong N, Schwarz CG, Botha H, Senjem ML, Spychalla AJ, Ahlskog JE, Knopman DS, Petersen RC, Jack CR, Jr. et al: MRI Outperforms [18F]AV-1451 PET as a Longitudinal Biomarker 
in Progressive Supranuclear Palsy. Mov Disord 2019, 34(1):105-113.

21. Hoglinger GU, Schope J, Stamelou M, Kassubek J, Del Ser T, Boxer AL, Wagenpfeil S, Huppertz HJ, Investigators AL, Tauros MRII et al: Longitudinal magnetic resonance imaging in progressive supranuclear palsy: A new combined score for clinical trials. Mov Disord 2017, 32(6):842-852.

22. Santacruz P UB, Litvan I, Grafman J. : Progressive supranuclear palsy: a survey of the disease course. . Neurology 1998, 50:1637-1647.

23. Whitwell JL, Lowe VJ, Tosakulwong N, Weigand SD, Senjem ML, Schwarz CG, Spychalla AJ, Petersen RC, Jack CR, Jr., Josephs KA: [(18) F]AV-1451 tau positron emission tomography in progressive supranuclear palsy. Mov Disord 2017, 32(1):124-133.

24. Golbe LI, Ohman-Strickland PA: A clinical rating scale for progressive supranuclear palsy. Brain 2007, 130(Pt 6):1552-1565.

25. ILitvan C, AMcKee,M Veiny,AParsa,KJellinger,LD'Olhaberriague, K Ray Chaudhuri, R K B Pearce: Natural history of progressive supranuclear palsy (Steele-Richardson-Olszewski syndrome) and clinicalpredictorsofsurvival:a clinicopathological study. J Neurol Neurosurg Psychiatry 1996, 60:615620.

26. Arena JE, Weigand SD, Whitwell JL, Hassan A, Eggers SD, Höglinger GU, Litvan I, Josephs KA: Progressive supranuclear palsy: progression and survival. Journal of Neurology 2015, 263(2):380389.

27. Yamamoto T, Tateno F, Sakakibara R, Furukawa S, Asahina M, Uchiyama T, Hirano S, Yamanaka Y, Fuse M, Koga $Y$ et al: Urinary Dysfunction in Progressive Supranuclear Palsy Compared with Other Parkinsonian Disorders. PLoS One 2016, 11(2):e0149278.

28. Respondek G, Hoglinger GU: The phenotypic spectrum of progressive supranuclear palsy. Parkinsonism Relat Disord 2016, 22 Suppl 1:S34-36.

29. Respondek G, Stamelou M, Kurz C, Ferguson LW, Rajput A, Chiu WZ, van Swieten JC, Troakes C, Al Sarraj S, Gelpi E et al: The phenotypic spectrum of progressive supranuclear palsy: a retrospective multicenter study of 100 definite cases. Mov Disord 2014, 29(14):1758-1766.

30. L. Donker Kaat MD AJWBMP, W. Kamphorst PhD, R. Ravid PhD, H.J. Duivenvoorden PhD, J.C. van Swieten MD PhD: Frontal presentation in progressive supranuclear palsy. Neurology 2007, 69:723729.

31. Cosseddu M, Benussi A, Gazzina S, Manes MA, Dell'Era V, Cristillo V, Turrone R, Alberici A, Borroni B: Natural history and predictors of survival in progressive supranuclear palsy. J Neurol Sci 2017, 382:105-107.

32. Koga S, Parks A, Kasanuki K, Sanchez-Contreras M, Baker MC, Josephs KA, Ahlskog JE, Uitti RJ, Graff-Radford N, van Gerpen JA et al: Cognitive impairment in progressive supranuclear palsy is associated with tau burden. Mov Disord 2017, 32(12):1772-1779.

\section{Tables}




\begin{tabular}{|c|c|c|c|}
\hline & $\mathrm{PSP} D \mathrm{~N}=59 \square$ & $\begin{array}{l}\text { PSP-RS } \\
\square N=43 \square\end{array}$ & $\begin{array}{l}\text { Non PSP-RS } \\
\square N=16 \square\end{array}$ \\
\hline Gender (Male/Female) & $34 / 25$ & $26 / 17$ & $8 / 8$ \\
\hline Age (years) & $64.7 \pm 8.0$ & $65.6 \pm 7.6$ & $62.4 \pm 8.7$ \\
\hline Age at onset & $62.0 \pm 2.3$ & $63.5 \pm 7.1$ & $57.9 \pm 9.6$ \\
\hline $\begin{array}{l}\text { The interval from the onset of symptoms to brain MRI } \\
\text { (years) }\end{array}$ & $3.8 \pm 2.3$ & $3.4 \pm 2.2$ & $4.7 \pm 2.2$ \\
\hline No. of death & 19 & 16 & 3 \\
\hline Median survival (years) & 12 & 12 & 15 \\
\hline No. of institutionalization & 31 & 22 & 9 \\
\hline Median time from onset to institutionalization (years) & 9 & 8 & 10 \\
\hline Early falls $\square \% \square$ & 69.5 & 86.0 & 25.0 \\
\hline Early FOG $\% \square$ & 30.5 & 30.2 & 31.3 \\
\hline Early bulbar paralysis (\%) & 66.1 & 79.1 & 31.3 \\
\hline Early apathy $\% \square$ & 35.4 & 44.2 & 12.5 \\
\hline MMSE & $23.0 \pm 5.1$ & $23.4 \pm 3.9$ & $21.9 \pm 7.8$ \\
\hline Early incontinence $₫ \% \square$ & 32.2 & 39.5 & 12.5 \\
\hline $\mathrm{M} / \mathrm{P}$ area ratio $\square \square$ & $21.3 \pm 7.1$ & $20.0 \pm 6.7$ & $24.3 \pm 7.6$ \\
\hline
\end{tabular}

Table 2. Predictors for early death 


\begin{tabular}{|c|c|c|c|c|}
\hline & \multicolumn{2}{|l|}{ Univariate } & \multicolumn{2}{|l|}{ Multivariate } \\
\hline & HR (95\%CI) & $P$ value & HR $(95 \% \mathrm{CI})$ & $\mathrm{P}$ value \\
\hline Gender $(\mathrm{M} / \mathrm{F})$ & $1.314(0.492-3.511)$ & 0.581 & & \\
\hline Age at onset & $0.999(0.942-1.060)$ & 0.970 & $2.087(1.146-3.801)$ & 0.016 \\
\hline phenotype $\llbracket$ non-RS $\square$ RS $\square$ & $0.417(0.118-1.477)$ & 0.417 & & \\
\hline Early falls & $3.554(1.012-12.485)$ & 0.048 & & \\
\hline Early FOG & $0.679(0.238-1.932)$ & 0.679 & & \\
\hline Early bulbar paralysis & $4.391(1.212-15.905)$ & 0.024 & & \\
\hline Early apathy & $3.040(1.128-8.189)$ & 0.028 & & \\
\hline Early incontinence & $4.958(1.704-14.430)$ & 0.003 & & \\
\hline MMSE & $0.886(0.755-1.040)$ & 0.139 & & \\
\hline $\mathrm{M} / \mathrm{P}$ area ratio $(\%){ }^{*}$ & $0.808(0.697-0.937)$ & 0.005 & $0.808(0.697-0.937)$ & 0.005 \\
\hline
\end{tabular}

*: adjusted for age and interval from onset to brain MRI

Table 3 Predictors for early institutionalization 


\begin{tabular}{|l|l|l|l|l|}
\hline & \multicolumn{2}{|l|}{ Univariate } & \multicolumn{2}{l|}{ Multivariate } \\
\hline & HR (95\%CI) & P value & HR (95\%CI) & P value \\
\hline Gender (M/F) & $1.716(0.783-3.765)$ & 0.178 & & \\
\hline Age at onset & $1.046(0.993-1.101)$ & 0.088 & $1.476(1.133-1.924)$ & 0.004 \\
\hline phenotype[non-RS[RS $\square$ & $0.626(0.274-1.430)$ & 0.266 & & \\
\hline Early falls & $1.678(0.760-3.705)$ & 0.200 & & \\
\hline Early FOG & $0.649(0.284-1.485)$ & 0.306 & & \\
\hline Early bulbar paralysis & $2.485(1.108-5.576)$ & 0.027 & & \\
\hline Early apathy & $2.153(1.021-4.536)$ & 0.044 & & \\
\hline Early incontinence & $3.174(1.369-7.360)$ & 0.007 & & \\
\hline MMSE & $0.928(0.812-1.061)$ & 0.279 & & \\
\hline M/P area ratio (\%) $*$ & $0.925(0.856-0.999)$ & 0.046 & $0.925(0.856-0.999)$ & 0.046 \\
\hline
\end{tabular}

*: adjusted for age and duration from onset to visit

\section{Figures}
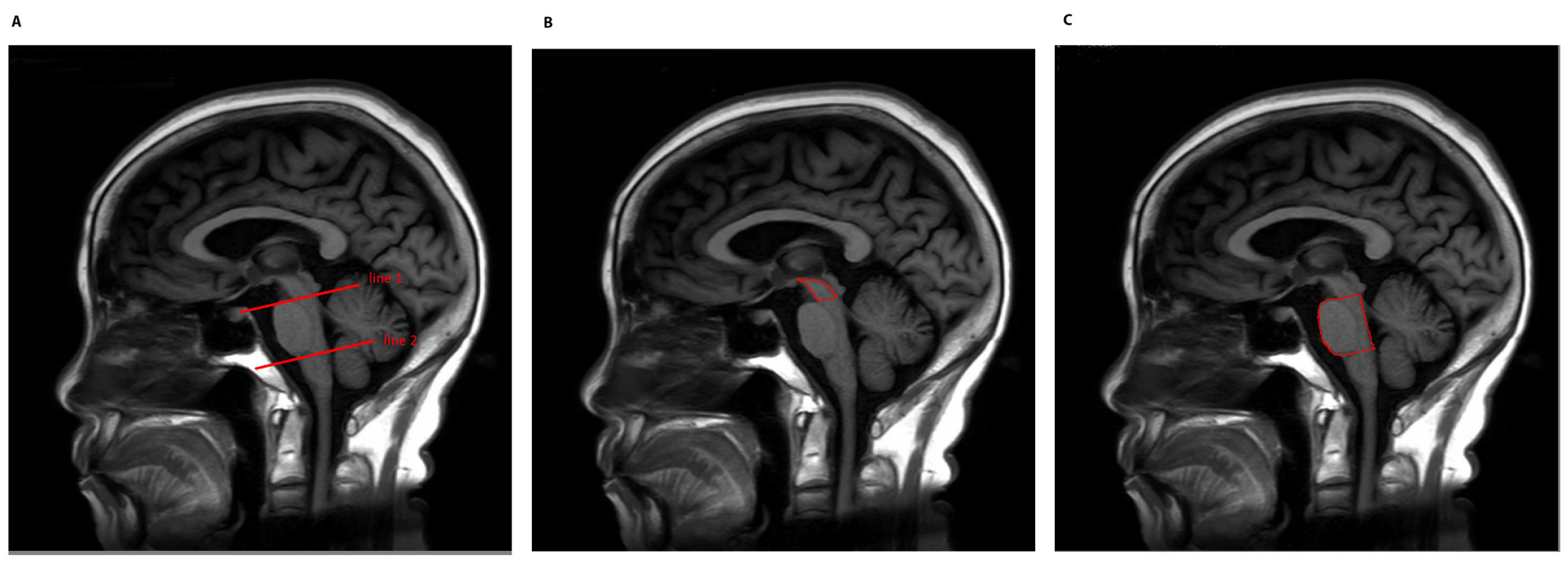

Figure 1

Sagittal T1-weighted MR images show midbrain area and pons area. (A) line 1 and line 2; (B) midbrain area; (C) pons area 